\title{
EL VIAJERO FRANQUISTA
}

\author{
JORGE CARRIÓN \\ Universidad Pompeu Fabra de Barcelona
}

\section{RESUMEN}

En la literatura española, el viaje ha estado siempre condicionado por la filosofía del espacio. Las concepciones del paisaje, el territorio y la historia firmadas por Unamuno, Ortega y Gasset o Eugenio d'Ors influyeron en la práctica del relato de viaje de sus contemporáneos y de los escritores viajeros posteriores. Esa constatación permite dividir la literatura de viajes de escritores españoles en la de los pro-espaciales (que defienden, explícita o implícita, de un territorio único, nacional y católico) y la de los contra-espaciales (que cuestionan esa perspectiva, que se apropió el franquismo). El análisis de algunos aspectos de la obra de Pemán, Díaz-Plaja, Aub o Gutiérrez Solana, entre otros, permite discutir la posibilidad de esa clasificación y reflexionar sobre la relación entre política y literatura durante el siglo XX.

Palabras clave: Literatura de viajes, Franquismo, Crítica espacial.

\section{THE PRO-FRANCO TRAVELER}

\section{ABSTRACT}

In Spanish literature, travelling has always been conditioned by philosophy of space. The interpretations of landscape, territory and history signed by Unamuno, Ortega y Gasset or Eugenio d'Ors influenced the way in which their contemporaries or later traveler writers narrated their travel stories. Such understanding allows to divide Spanish travel writers in pro-spacial writers (they defend, in both explicit or implicit ways, a unique, national and catholic territory) and counter-spacial writers (they argue about this same perspective). The analysis of some aspects of Pemán's, Díaz-Plaja's, Aub's or Gutiérrez Solana's works, among others, allows a discussion on the possibility of this classification and a reflection on the relationship between Politics and Literature during the 20th century.

Key Words: Travel Literature, Francoism, Spatial Criticism.

\section{VIAJE Y ESPACIO}

El viaje es una práctica del espacio. Como nos enseñaron el romanticismo y el surrealismo, también es una forma de politizar o de subvertir el espacio; es decir, de crear una reafirmación o una tensión ideológicas entre el arte y la configuración institucional del espacio nacional o, a menor 
escala, del urbano. La lealtad de gran parte de los poetas románticos hacia los ideales nacionalistas, a cuyos programas contribuyeron mediante la creación de un archivo poético que trató de fijar la relación entre paisaje y folklore, permite afirmar que la nación europea se construye en un proceso de textualización en que participan —en paralelo- políticos, militares y literatos. La idea de revolución que es inherente al arte surrealista y a su lectura benjaminiana permite observar la relación entre arte y espacio político desde el punto de vista contrario: la poesía como estrategia de desarticulación del poder imperante, de cuestionamiento de las formas social y políticamente habituales de praxis urbana. A favor o en contra del espacio político que se recorre: entre esos dos extremos si sitúan, conscientemente o no, los escritores de viaje ${ }^{1}$.

Es imposible emancipar el viaje del contexto político en que tiene lugar. Durante el primer tercio del siglo XX, los gobiernos europeos se percataron del potencial propagandístico del turismo; sobre todo los dictatoriales. Tanto la Alemania nazi (que produjo, por ejemplo, una impresionante escenografía durante los Juegos Olímpicos de Berlín de 1936 con el objeto de convencer a los visitantes de las bondades y el poder del III Reich) como la Unión Soviética (donde se creó la agencia de viajes Intourist para promover el bolchevismo) trabajaron conscientemente en el viaje como instrumento de propaganda. Como ha estudiado Shasha D. Pack, también el recién configurado gobierno de Francisco Franco participó de esa tendencia internacional:

El 7 de junio de 1938, durante una breve pausa de los enfrentamientos antes de la decisiva batalla del Ebro, el ministro del Interior, Ramón Serrano Suñer, anunció que las principales carreteras de la zona nacional en el norte se abrirían a los viajes turísticos organizados, para que los visitantes extranjeros y españoles pudieran 'observar la tranquilidad y el orden de las regiones recién conquistadas por nuestras armas' (...) Durante los primeros años años de la Guerra Civil, Bolín trabajó como guía de los periodistas extranjeros que visitaban el campo de batalla nacional. Su viajes organizados, llamados Rutas de Guerra, operaban más o menos del mismo modo: se llevaba a sus clientes en autobús a las zonas de combate, narrándoles los hechos de forma muy politizada ${ }^{2}$.

Durante ese mismo primer tercio del siglo XX, la literatura de derechas europea sintoniza, en líneas generales, con una codificación nacionalista del territorio y de su paisaje. En el caso español, según el falangismo y el franquismo, el espacio nacional se define por su unidad: entre los miembros de la familia; entre el ciudadano y la Iglesia Católica; entre el hombre español y el suelo en que habita. La propia idea de España es para

\footnotetext{
${ }^{1}$ CARRIÓN, Jorge. Viaje contra espacio. Juan Goytisolo y W.G. Sebald. Madrid: Iberoamericana. 2009.

${ }^{2}$ PACK, Sasha D. La invasión pacífica. Los turistas y la España de Franco. Ana Marí (trad.). Madrid: Taurus, 2009, pp. 61-62.
} 
Primo de Rivera unidad de destino. Esa unidad, obviamente, suprime la pluralidad de lenguas, credos $\mathrm{u}$ orientaciones sexuales propia de cualquier comunidad moderna. También crea un ámbito de opinión en que no se contempla la posibilidad de la disensión o de la discrepancia. El espacio totalitario. La unificación de ese espacio fue expresada así por el escritor falangista Ernesto Giménez Caballero en 1928:

Desde luego, tiene razón Ortega y Gasset, al soñar que son precisas todas las divergencias previas, todos los regionalismos preliminares, todos los separatismos — sin asustarnos de esta palabra-, para poder tener un verdadero día el nodo central, un motivo de hacinamiento, de fascismo hispánico ${ }^{3}$.

Efectivamente, Ortega y Gasset publicó a inicios del siglo XX textos que no sólo alimentaron el discurso nacionalista falangista, sino que condicionaron la filosofía del desplazamiento en las instituciones académicas españolas de la época. En un artículo titulado «La pedagogía del paisaje» (El Imparcial, 17 de septiembre de 1906), por ejemplo, unía la mística, la geografía y la religión, con la voluntad de transmitir «moral e historia, dos disciplinas de exaltación que nos hacen no poca falta a los españoles» ${ }^{4}$. Poco después, en 1914, con la publicación de Vieja y nueva política, Ortega brindó a la Falange parte de sus parámetros políticos y de su vocabulario y retórica, al tiempo que estableció las líneas generales de la relación entre viaje peninsular y evangelización fraternal: «vamos a ver España y a sembrarla de amor y de indignación. Vamos a recorrer los campos en apostólica algarada ${ }^{5}$. Para entonces, la Institución Libre de Enseñanza ya había impuesto la pedagogía de la excursión, cuyos objetivos eran «la comunión con la Naturaleza», «la doctrina estética del paisaje», «la educación al aire libre», «la religiosidad natural» ${ }^{6}$.

Sus contemporáneos trabajaron en la misma dirección. La ruta de don Quijote (1905), de Azorín, comienza con la constatación en primera persona de que él tiene «que realizar una misión sobre la tierra» y que de su libro debe desprenderse una «visión neta y profunda de la España castiza» ${ }^{7}$. Ocho años más tarde, el mismo autor escribió el artículo «La conquista de España», donde se lee: «la conquista de España entera está por realizar» ${ }^{8}$. Una conquista narrativa, una textualización del territorio que se expresa

${ }^{3}$ Cit. por CARBAJOSA, Mónica, y CARBAJOSA, Pablo. La corte literaria de José Antonio. La primera generación cultural de la Falange. Madrid: Crítica, 2003, p. 53.

${ }^{4}$ ORTEGA Y GASSET, José. Obras Completas I (1902-1916). Madrid: Revista de Occidente, 1946, p. 57.

${ }^{5}$ Cit. por CARBAJOSA, Mónica, y CARBAJOSA, Pablo, op. cit, p. 53

${ }^{6}$ ORTEGA CANTERO, Nicolás. «La experiencia viajera en la Institución Libre de Enseñanza». En: VV.AA. Viajeros y paisajes. Madrid: Alianza Universidad, 1988, pp. 67-77.

7 AZORÍN. Obras completas II. Madrid: M. Aguilar Editor: 1947, pp. 242-243.

${ }^{8}$ Ibid., 799. 
en términos de «misión» y de «conquista». Después de hablar sobre guerras coloniales, Unamuno escribe en un artículo publicado por La Laguna de Tenerife en agosto de 1909, en sintonía con un regeneracionismo que no está reñido con el negacionismo: «Y es, sin embargo, merced a esto como ha podido asentarse el reinado de la razón y de Cristo (...), el español casi nunca ha exterminado las razas indígenas de aquellos pueblos que ha conquistado» ${ }^{9}$. En 1939, a su regreso de París, Azorín escribió «En España», donde se incita a asociar la lectura de los clásicos con la eternidad de lo español en Europa y en América, con la intención evidente de concordar con el ánimo neoimperialista del franquismo recién triunfante.

Esas líneas de convergencia con la ideología de los vencedores certifican una dirección única de la literatura de viajes en castellano: la que se configura en Giner de los Ríos, atraviesa los paisajes de Unamuno, Azorín, Ortega y Gasset, Pemán o Eugenio d'Ors y, tras la guerra civil, sigue insistiendo en la obra de esos autores y otros afines o se recicla en los libros de viaje de la nueva generación, con Camilo José Cela al frente.

\section{HACIA UNA DEFINICIÓN DEL VIAJERO FRANQUISTA}

Eugenio d'Ors puede ser considerado arquetípico en tanto que escritor viajero franquista - la noción que en estas líneas propongo, pese a su reduccionismo y sus problemas intrínsecos, que a nadie se le escapan. Esa faceta del autor de Glosario fue destacada, entre otros, por Guillermo DíazPlaja: «El viaje fue en Eugenio d'Ors una categoría definitoria», escribe. En la literatura moderna y contemporánea, la alteridad, el fantasma es siempre el turista; de modo que en su ensayo Díaz-Plaja quiere distanciar al protagonista de su semblanza del turismo, y para ello utiliza dos conceptos que son fundamentales para entender el viaje franquista: «Dos motes, sin embargo, absuelven a este viajero tenacísimo de cualquier acusación de banalidad: el mote de Misión y su autocalificación de Católico Errante ${ }^{10}$. La poética del viaje es, por tanto, una política y una ética. Voluntad misionera de acercamiento al prójimo para evangelizarlo, dentro del contexto de «las fronteras de la Catolicidad», como recuperación de una tradición de conquista física y espiritual claramente neoimperialista.

Es conocida la recuperación que hizo el franquismo de la estética y de la retórica del imperialismo español de los siglos XVI y XVII. El 1 de julio de 1937, en un discurso pronunciado en Burgos, por ejemplo, Francisco Franco dijo lo siguiente: «Es la lucha en defensa de Europa y, una

\footnotetext{
${ }^{9}$ UNAMUNO, Miguel de. Obras completas I. Paisajes y ensayos. Madrid: Escelicer, 1966, p. 325.

${ }^{10}$ DÍAZ -PLAJA, Guillermo. Ensayos elegidos. Madrid: Ed. de la Revista de Occidente, 1965, p. 413.
} 
vez más, cabe a los españoles la gloria de llevar en la punta de sus bayonetas la defensa de la civilización, de mantener una cultura cristiana y de mantenerla al estilo de Don Quijote» ${ }^{11}$. La alusión al personaje de Cervantes no es casual: el falangismo y el franquismo se apropiaron del capital simbólico más importante de la tradición cultural española. El Escorial, Carlos I y Felipe II, los poetas místicos, la literatura del Siglo de Oro, Velázquez, El Greco: ése es el banco del que se nutre la quimera imperialista del Generalísimo. El archivo de los iconos de la época en que España era una potencia europea y una metrópolis que dominaba la mayor parte de América. El supuesto europeísmo entronca, por tanto, con una defensa de la cristiandad que amplía las fronteras de «lo hispánico» a América Latina, en la línea de la Hispanidad nacida con el cambio de siglo y que había sido impulsada por Primo de Rivera. Los valores de esa ampliación son el paternalismo postcolonial: una reformulación de la conquista misionera. Cuando en 1953 Camilo José Cela regresa de su viaje a Colombia, Ecuador y Venezuela, el director general de Prensa le saluda en las páginas de El español como al «nuevo arquetipo de misionero civil de la España de Francisco Franco» ${ }^{12}$.

El propio Díaz-Plaja, en su autobiografía Retrato de un escritor, explica que el viaje le interesó siempre para «ganar amigos» y a causa de «un ánimo religioso que queda saturado por la hermosura del mundo» ${ }^{13}$. Es decir, más allá de la adscripción concreta a una religión, la católica en este caso, el viaje franquista difumina sus contornos mediante fórmulas panteístas, sin abandonar la premisa de «amar al prójimo». En un texto medular, titulado justamente «Teoría del viaje», Díaz-Plaja argumenta una terapéutica y una pedagogía del desplazamiento, a partir de la historia del viaje, que ha desembocado - según él- en el turismo como una «necesidad anímica», que empatiza con la necesidad de «fraternizar»:

No se ha observado bastante el valor de fraternización que el viaje trae aparejado, ya que de la exploración, siquiera superficial, que al viajar realizamos, surge una educación hecha del hábito de comprender actitudes que no son las que frecuentamos habitualmente. Así, el turismo, sería una unesco, de apariencia más frívola $\mathrm{y}$ de ademanes menos protocolarios, pero de indubitable fuera educativa ${ }^{14}$.

El «amor al prójimo» de Díaz-Plaja sintoniza con el «amor» que encontramos en varios títulos topográficos de Giménez Caballero: Amor a Cataluña (1942), Amor a Andalucía (1943), Amor a Galicia (1947), Amor a

${ }^{11}$ DEL RÍO CISNEROS, Agustín (ed.). El pensamiento político de Franco. Antología. Madrid: Servicio Informativo Español, 1964, p. 35.

12 GUERRERO, Gustavo. Historia de un encargo: «La catira» de Camilo José Cela. Barcelona: Anagrama, 2008, p. 23.

${ }^{13}$ DÍAZ-PLAJA, Guillermo. Retrato de un escritor. Barcelona: Pomaire, 1978, p.265.

${ }^{14}$ DÍAZ-PLAJA, Guillermo. Ensayos elegidos. Madrid: Ed. de la Revista de Occidente, 1965, p. 526. 
Argentina (1948), Amor a México (1948), Amor a Portugal (1950); no en vano estamos ante el autor de La nueva catolicidad (1933) y Roma Madre (1939), donde leemos que lo que él siempre hizo con lo que había aprendido era comunicarlo «con ansia dominicana, de fraile mendicante y misionero» ${ }^{15}$. El mencionado ensayo de Díaz-Plaja prosigue con una defensa del viaje como modo de abolición de fronteras y, por tanto, de superación de los nacionalismos europeos que a tanto horror condujeron el siglo pasado. Díaz-Plaja se sitúa de ese modo en una posición de disidencia parcial dentro del régimen político en que desarrollaba su vida académica. La filosofía política de José María Pemán, en cambio, obviamente vinculada con una concepción espacio-temporal de signo fascista, se resume en su forma de ordenar los epígrafes de un libro temprano: En la hora de la dictadura (1923-1930): «La Patria en el espacio: sociedad natural» y «La Patria en el tiempo: tradición». En el contexto local los conceptos se inscriben en el regeneracionismo de la dictadura de Primo de Rivera; el contexto europeo, en cambio, obliga a verlos en relación con la emergencia, si no consolidación, de las corrientes filosóficas racistas y nacionalistas que configuraron el caldo de cultivo del nazismo. Como Eugenio d'Ors, Pemán viajó largamente por América y su voluntad de evangelización cultural y la solidez de sus convicciones ideológicas le negaron la posibilidad de viajar realmente, si el viaje significa cambio y no reafirmación sin grietas. Sus viajes americanos siempre tuvieron como razón de ser una conferencia o una embajada política, lo que condicionó el tono de sus intervenciones públicas, que se pueden resumir en títulos como «Mensaje a la América española» (3 de junio de 1941) o en afirmaciones como que él sólo respondía a «mi Fe, mi Patria y mi Arte» ${ }^{16}$. Existe, por tanto, una relación - una unidad - consciente entre el viajero en el extranjero y el estado que lo espera y unas obligaciones derivadas de ese vínculo. Lo que Camilo José Cela llamó «mi deber de español» en referencia a su «misión civil» en América ${ }^{17}$. Lo que yo llamo un ánimo pro-espacial, esto es, de reafirmación textual de la ideología dominante proyectada sobre una concepción del espacio.

Esas obligaciones tienen que ver con el hecho de que la dictadura franquista subvencionara gran parte de los viajes de intelectuales españoles por América. El programa «La voz de España en América», del Instituto de Cultura Hispánica en América, patrocinó las giras de escritores como Laín Entralgo, Antonio Tovar, D’Ors, Díaz-Plaja, Giménez Caballero, Eugenio Montes, Pemán, Dámaso Alonso, Panero, Rosales, Foxá, etcétera ${ }^{18}$. La agen-

\footnotetext{
${ }^{15}$ Cit. por CARBAJOSA, Mónica, y CARBAJOSA, Pablo. op. cit., p. 223.

${ }^{16}$ PEMÁN, José María. Narraciones y ensayos. Obras Completas II. Madrid: Escelicer, 1947.

${ }^{17}$ GUERRERO, Gustavo, op. cit., p. 29.

${ }^{18}$ Ibid., 178.
} 
da de conferencias y encuentros diplomáticos, dictada por los intereses de la política internacional y de la propaganda franquistas, minaba obviamente la posibilidad del encuentro con el otro y con el azar que impulsa el viaje. Se opera así una transformación: de lo individual a lo colectivo, de lo personal a lo institucional, de la poética literaria al programa de estado. De la literatura a la geopolítica. Los escritores continúan produciendo textos durante sus viajes: artículos, conferencias, proclamas, mensajes, sermones, diálogos, diarios, cartas. Estos pueden ser leídos en primer término como piezas de la obra individual, pero remiten por sus características comunes no sólo a una época, sino también a un horizonte estratégico, religioso y político. No hay más que observar las fronteras que limitan los desplazamientos de los escritores franquistas. Fronteras profesionales: la reducida oferta de embajadas y corresponsalías interesantes o ojos de un nacional-católico. Fronteras religiosas: los países cristianos. Sánchez Mazas fue corresponsal de $A B C$ y agregado cultural en la Roma de los años 20; Giménez Caballero ejerció en esa época como profesor en Estrasburgo, y en los 60 fue embajador en Paraguay y Brasil; Dionisio Ridruejo combatió en Rusia en las filas de la División Azul; Eugenio d'Ors vivió varios años del periodismo en París; Eugenio Montes fue el corresponsal de $A B C$ en Alemania durante el nazismo y reunió sus crónicas centro-europeas en El viajero y su sombra (1940); Jacinto Miquelarena tuvo corresponsalías de $A B C$ en Berlín y París. Las excursiones latinoamericanas fueron excepciones de una topografía esencialmente europea.

Una de las pocas fracturas de ese consenso geopolítico se dio con nuestro vecino africano y tuvo lugar durante la Guerra de Marruecos y en los años que la separaron de la Guerra Civil. Sánchez Mazas escribió crónicas sobre la campaña militar; Giménez Caballero publicó Notas marruecas de un soldado (1924). La perspectiva colonial es evidente en esos textos, que defienden el espíritu civilizador de la intervención española en Marruecos. Pese a una tendencia general a defender a ultranza el pasado nacional-católico español, en detrimento del pasado árabe ibérico, hubo destacados intelectuales franquistas que consideraron el periodo islámico como una parte importante de nuestra historia y que expresaron esa convicción a través de textos de viaje. Tal es el caso de Pemán, que unió simbólicamente la conquista y la colonización de América de los siglos XVI y siguientes con la colonización de las Filipinas o Marruecos en una oración memorable: «Esa fineza colonizadora española, mestizaje y muzarabismo espiritual, que es una de las obras maestras de la civilización humana ${ }^{19}$. Pemán, autor del himno nacional, católico e incluso monárquico antes de la guerra, que se definió a sí mismo como alguien «por temperamento y por formación

${ }^{19}$ PEMÁN, José María. Doctrina y oratoria. Obras Completas V. Madrid: Escelicer, 1947, p. 489. 
clásica, un fervoroso creyente del orden y de la jerarquía en todas las cosas» (Pemán 1947: 47) ${ }^{20}$, viajó a Tánger antes de la guerra civil con motivo de la Fiesta de la Raza y en su relato, titulado «Apuntes de una excursión a Tánger», hizo hincapié en la inmovilidad del árabe, en su atraso expresado en la metáfora del retraso del reloj (íbid., 727). Años más tarde, él mismo supo releer esa desventaja como una virtud, cuando para justificar la presencia de tropas árabes junto con las de Francisco Franco escribió el artículo «Los moros amigos» reconsideró la invasión islámica de la Península Ibérica como algo positivo, porque permitió que España tuviera el papel de «guardianes de la cultura clásica» (Pemán 1947b: 488). Tantos siglos más tarde, el autor de la novela satírica De Madrid a Oviedo, pasando por Las Azores (1933) ve con simpatía el desfile de los «moros amigos» por ser un eco, a sus ojos, de otro momento histórico en que también le tocaba a España asumir el rol de defensor de la cultura clásica.

Una cultura europea, mediterránea, bíblica. La que se discutió políticamente durante la República al más alto nivel, y en términos de viaje. Pemán, en un artículo titulado «Mediterráneo» atacó el proyecto de Fernando de los Ríos de un viaje de estudios para estudiantes universitarios por el Mare Nostrum, arguyendo que la pluralidad mediterránea que tal viaje pedagógico busca comprender no existe: «comprenderán que no hay tales relativismos y pluralidades enervadoras: que Roma, Atenas, Jerusalén y sus reflejos alejandrinos, tunecinos y argelinos de la orilla Sur, forman un círculo cerrado, donde se inserta toda la verdadera cultura» (Pemán 147b: 213). Contra esa idea de cerrazón se gestó finalmente el célebre viaje de junio y julio de 1933 que durante cuarenta y cinco días recorrió el Mediterráneo con profesores y alumnos de la órbita republicana a bordo del Ciudad de Cádiz (cuyo nombre fue Infanta Isabel hasta la llegada de la República y que fue hundido por un submarino italiano en 1937). Los diarios conservadores acusaron al gobierno de Azaña de malversación de fondos y de elitismo intelectual, prosiguiendo con una actitud de cuestionamiento del proyecto que había comenzado desde el momento en que este fue debatido en las Cortes (Gracia Alonso 2006).

Esa disputa es fundamental. Enfrenta los dos modelos de viaje. Dos modelos posibles, discutibles, de ningún modo absolutos, porque la cultura literaria española durante los tres primeros cuartos del siglo XX está llena de cruces y de ambigüedades. Según sabemos por una carta de Zenobia Camprubí fechada el 9 de agosto de 1948, los Jiménez y los Pemán cenaron en Buenos Aires, justo antes de que el escritor franquista y su esposa partieran de regreso, hacia Lisboa (Camprubí 699). Los intercambios epistolares y las relaciones de amistad fueron frecuentes entre los que

${ }^{20}$ PEMÁN, José María. Doctrina y oratoria. Obras Completas V. Madrid: Escelicer, 1947.

Revista de Literatura, 2011, enero-junio, vol. LXXIII, n. ${ }^{\circ}$ 145, 269-282, ISSN: 0034-849X 
se fueron y los que se quedaron, entre los exiliados republicanos y los altos cargos de las instituciones culturales franquistas. No obstante, merece la pena definir el «viaje republicano».

\section{3. ¿EL VIAJERO REPUBLICANO?}

Hemos partido — a sabiendas - de una hipótesis reduccionista: durante al menos los dos primeros tercios del siglo XX existen en España dos formas opuestas de viajar, de izquierdas y de derechas. Soy consciente de que los matices son infinitos, de que las prácticas del viaje son al cabo personales, que esas tendencias políticas se pueden fragmentar exponencialmente. Sin embargo, me parece ciertamente estimulante observar la relación entre literatura y política desde la intersección que otorga el viaje y tratar de observar en él una oposición entre viajeros de izquierdas y viajeros de derechas. Es decir, si como ha escrito Adrien Pasquali las oposiciones clásicas son la de viajero que escribe versus escritor que viaja, la de viajero versus erudito «de chambre» y la de viajero versus turista, tres manifestaciones de una misma e inacabable «querelle sur la manière de voyager» (Pasquali 1994: 31), puede resultar fértil ampliar esa misma disputa mediante una dicotomía nueva: viajeros de izquierdas y de derechas, lo que en el contexto español contemporáneo podría ser —reduciendo, insistoviajeros republicanos y viajeros franquistas. La hipótesis quiere ser una pregunta de imposible respuesta única: es decir, una manera de cuestionar algunas poéticas relacionadas con prácticas del viaje, en un marco histórico que supuestamente se puede dividir en dos marcos ideológicos, para tratar de entender mejor la importancia de los desplazamientos en la literatura española contemporánea.

En principio, el viaje republicano se definiría por la ausencia de ese ánimo evangelizador que se ha visto en el viaje franquista. Por la ausencia del mote de misión. Sin embargo, enseguida nos encontramos la primera paradoja: el emblema de la pedagogía republicana es «la misión pedagógica». Creadas en 1931 para remediar la desigualdad cultural entre las ciudades y las aldeas, bajo el principio de igualdad de todos los ciudadanos y por tanto de justicia social, consistió en la circulación por España de bibliotecas, museos, compañías teatrales o coros itinerantes, cuya razón de ser era el acercamiento de la cultura metropolitana a la periferia rural. Sin embargo, Manuel B. Cossío, presidente del Patronato de Misiones Pedagógicas y su principal impulsor, matizó que las misiones pedagógicas no tenían como objetivo el adoctrinamiento. Ahí radicaría una primera diferencia entre las dos formas de entender el viaje: el republicano rechazaría conscientemente la posibilidad de la conquista.

La segunda - y fundamental - radica en las circunstancias históricas en que el viaje republicano fue interrumpido como posibilidad práctica. 
La exploración de la alteridad (lo afroamericano), el cuestionamiento de lo propio (el catolicismo) y el reconocimiento de lo íntimo (la homosexualidad) que Lorca llevó a cabo entre 1929 y 1930 en Poeta en Nueva York, un poemario con voluntad de diálogo transatlántico, geográfico y poético (piénsese, por ejemplo, en las concomitancias literarias y políticas con la obra contemporánea de Pablo Neruda), constituyen las líneas mayores de una poética del viaje que fue cercenada por el asesinato político. Más allá —incluso- de eso, el trauma de la derrota en la guerra civil implicó la suspensión de la posibilidad de la literatura de viajes en la literatura española de mayor calidad ética y estética. Los escritores del exilio no pudieron encontrar, por lo general, estrategias para abordar libros de viaje que superaran su condición de exiliados. Esa incomodidad se traduce, en el caso ejemplar de Juan Ramón Jiménez, en una distancia abismal entre sus dos principales obras de viaje. En Diario de un poeta recién casado (1917), la estructura espacial y temporal es cristalina, ordenada, consciente; el viaje evidencia una búsqueda y un encuentro, en una de las formas clásicas de la literatura de viajes: el cuaderno de bitácora. Guerra en España, en cambio, reúne textos escritos en lugares diversos entre 1936 y 1953, que fueron encontrados por Ángel Crespo en la Biblioteca de la Universidad de Puerto Rico dispuestos en tres sobres; su primera parte, «Desterrado (Diario poético)», es el reverso espacial y poético del libro de 1917, la evidencia de la imposibilidad de escribir literatura de viajes similar a la anterior a 1936. Por cierta inercia crítica, se acostumbra a citar La gallina ciega (1971), el diario español de Max Aub, como el libro de viaje canónico de la literatura del exilio. Sin embargo, pese a su calidad, no deja de ser una obra convencional en sus planteamientos narrativos y que, por enésima vez, sucede en la Península Ibérica. La literatura de viajes de Aub fue mucho más audaz en algunos pasajes de sus diarios internacionales o incluso en su biografía apócrifa de Jusep Torres Campalans (1958), donde se crea un puente desafiante entre Barcelona, París y Chiapas.

La literatura de viajes trabaja fundamentalmente en el tiempo presente; la literatura del exilio, en cambio, es sobre todo evocativa. Escribre Ricardo Gullón, en el diario de una excursión por el Caribe: «Desde Naguabo estamos recordando tangos del cercano ayer. Después son las canciones populares de nuestra tierra; unos y otras cantan en la memoria y la excitan avivando la nostalgia» (Gullón 69). En ese conflicto entre los desplazamientos del presente y el trasfondo de la memoria se sitúa la literatura de viajes escrita por autores exiliados. La topografía que en el presente sería vista como símbolo del poder dictatorial, en el recuerdo es contemplada como símbolo de la patria perdida. El caso de Luis Cernuda es paradigmático al respecto. Se exilió por motivos políticos y cultivó en su última etapa vital una poesía extremadamente crítica con la historia y la realidad españolas, pero transformó la guerra civil en mito bíblico y des- 
tacó exaltadamente tres símbolos claramente imperialistas: el Escorial, Felipe II y la «epopeya de la Conquista». «Lo que se sabe de la historia de Cernuda como hombre no permite catalogarlo definitivamente ni como revolucionario ni como reaccionario», escribe Bernard Sicot; y añade: «en el contexto español de entonces, igual que en el del exilio, escapa a la ortodoxia de los discursos oficiales, de izquierdas como de derechas, para llevar a cabo una especie de reconquista de las palabras, de la historia y, a través de los símbolos, del imaginario nacional» (Sicot 2003: 209- 221). Los escritores de izquierda y los de derecha comparten el capital simbólico de la época imperial española. Es más: los discursos se entreveran, las prácticas literarias y espaciales se entrecruzan, «lo español» se confunde en una maraña de disputas por apropiárselo y re-semantizarlo. Escritores de España y del Exilio comparten la voluntad de resucitar, mediante estrategias a menudo divergentes, cierto espíritu nacional que ha desparecido: «El nacionalismo español se ha alimentado históricamente del trabajo de pensadores tanto progresistas como conservadores» (Balibrea 2007: 58).

Tal vez el mejor ejemplo de escritor de viajes español que durante el primer tercio del siglo XX llevó a cabo un proyecto indudablemente antirreligioso y antiesencialista, sin asomo de intenciones misioneras o fraternales, sin voluntad de conquista del espacio, sea el de José Gutiérrez-Solana. Fijémonos, por tanto, en sus crónicas de viaje, escritas durante las dos primeras décadas y publicadas en el volumen La España negra (1920). Fijémonos, por ejemplo, en su topografía: pueblos y ciudades como Calatayud, Santoña, Valladolid, Medina del Campo, Oropesa o Ávila; y dentro de ellos: la cárcel, el hospital, los cafés, las audiencias, el hospital psiquiátrico, la feria. Es decir: una topografía que a priori parecería anti-turística, que pretende introducirse en la España verdadera y no reproducir meramente la fachada legitimada por el pedigrí histórico. La lectura de la obra de Gutiérrez-Solana, no obstante, nos lleva más lejos. Si observamos, por ejemplo, cómo procede durante su visita a la casa de Santa Teresa, en Ávila, un lugar que todavía está abierto al público y todavía exhibe los mismos objetos que entonces:

En el convento de Carmelitas Descalzos hay una habitación dedicada a los recuerdos de Teresa de Jesús. Son éstos relicarios de plata, en los que pude ver un dedo repugnante, rodeado de cabellos de la Santa, unas disciplinas muy apolilladas por el tiempo, y una cosa que me dijeron que era el corazón y que pude ver a través de un cristal, y un pie negro y amojamado que parecía de momia. Lo que ponía un sello de poesía a todas estas porquerías y piltrafas de ultratumba era el jardín de al lado; un jardín conventual y abandonado en que la Santa se distraía, en los ratos de ocio, en cavar la tierra y plantar flores. (Gutiérrez Solana 2000: 156).

La desacralización es absoluta. Aunque el título del capítulo es «La casa de Santa Teresa», el párrafo se inicia con su transformación en una mu- 
jer: «Teresa de Jesús». Lo mismo ocurre con sus posesiones: de reliquias en potencia, a causa de su contenedor («relicarios de plata»), son nombradas en su realidad material: «porquerías y piltrafas». La alusión final al ocio del personaje histórico culmina esa visión absolutamente humana de una personalidad vinculada con el Siglo de Oro, es decir, con la supuesta gloria política y espiritual española. Algunos capítulos antes, otra escena memorable ha mostrado la esencia de la iglesia contemporánea — desde el punto de vista del narrador Gutiérrez-Solana-, en una «de las calles más típicas de Medina del Campo»:

donde hay conventos de frailes descalzos. Éstos son tan holgazanes, que se levantan de la cama por la tarde; todo el día se lo pasan durmiendo y comiendo (...). Enfrenten están las casa de las mujeres de la mala vida, que les llaman mucho desde la calle; pero ellos no las hacen caso, porque para estos menesteres tiene la comunidad mejores mujeres entre las monjas. Anochecido, los cagones del pueblo, que salen de las casas de lenocino, se ponen en fila, y bajándose las bragas, con las posaderas al aire, hacen del cuerpo bajo las rejas del convento; los frailes, que a esa hora suelen estar borrachos, se asoman por las ventanas y vomitan en las espaldas de los cagones y vuelvan sus pestilentes bacines. (íbid., 110).

Lo «típico», por tanto, según la estética deformante del escritor y pintor, no es el monumento, la calle barroca o el panteón de las glorias nacionales, sino lo esencial de la cultura española. Y esa esencia se encuentra en la periferia de la sociedad: en los lugares de castigo donde pudren su condena los anarquistas, en la puerta de la plaza de toros donde se desangra al animal muerto, en el burdel; se encuentra también en las tradiciones populares, como las corridas, los entierros o las procesiones religiosas; y, por último, en el propio centro de la sociedad, pero oculta tras las paredes de las instituciones o tras las ficciones religiosas o políticas. En Medina del Campo, cerca de los conventos que para Gutiérrez-Solana son sinónimos de la escatología española, está la Audiencia, cuyo presidente es un «viejo carcamal», un «pajarraco, calvo y con patillas blancas» que «está pidiendo un ataúd muy largo y estrecho» (íbid., 109). Los funcionarios, los políticos, los curas, los monjes y las monjas son objeto de la deformación sistemática de Gutiérrez-Solana. Sin caer nunca en el lenguaje de la propaganda, sin articular un discurso ideológicamente pornográfico, el modo como su viaje por España enfoca la dimensión más atávica del país y la retrata conduce a la constatación de que el narrador ha creado un artilugio estético de intención anti-clerical. Un artefacto político en el mejor sentido del término: actividad del ciudadano cuando interviene en los asuntos públicos con su opinión. Un artefacto político, en fin, que se sabe consciente de la extraña tradición artística en que se insiere: la de La España negra (1899) de Emile Verhaeren y Darío de Regoyos, tradición híbrida, pictórica y literaria, furiosa, «buscábamos algo nuevo y 
distinto de lo que ambicionan los ingleses que en sus viajes no buscan más que el confort» (Verhaeren y Regoyos 1963: 21).

¿Se puede decir que Gutiérrez-Solana es un viajero republicano? No lo creo. Sí, en cambio, considero que su proyecto se inscribe en la franja más ancha del viaje de izquierdas, en lo que tiene de anti-clerical, de crítico, de contra-espacial. A grandes rasgos, al menos en el caso español, el viaje de derechas se corresponde con el de aquellos que viajan a favor del espacio político nacional-católico; el de izquierdas estaría protagonizado por quienes lo hacen en contra de ese mismo espacio. Ampliando las dicotomías de Pasquali con que empezaban estas líneas: los viajeros pro-espaciales versus los viajeros contra-espaciales. Ninguno de los dos, como se ha visto, está exento de contradicciones y dificultades de lectura. He intentado trazar las líneas horizontales y verticales en que se inscriben algunos de los proyectos de esos viajeros, en el caso español, durante los dos primeros tercios del pasado siglo. Un radar posible, en el que seguir observando puntos móviles.

\section{REFERENCIAS BIBLIOGRÁFICAS}

AZORÍN. Obras completas II. Madrid: M. Aguilar Editor, 1947.

BALIBREA, Mari Paz. Tiempo de exilio. Mataró: Montesinos, 2007.

CAMPRUBÍ, Zenobia. Epistolario I. Cartas a Juan Guerrero Ruiz 1917-1956. Madrid: Publicaciones de la Residencia de Estudiantes, 2006.

CARBAJOSA, Mónica, y CARBAJOSA, Pablo. La corte literaria de José Antonio. La primera generación cultural de la Falange. Madrid: Crítica, 2003.

CARRIÓN, Jorge. Viaje contra espacio. Juan Goytisolo y W.G. Sebald. Madrid: Iberoamericana, 2009.

DÍAZ-PLAJA, Guillermo. Ensayos elegidos. Madrid: Ed. de la Revista de Occidente, 1965.

-. Retrato de un escritor. Barcelona: Pomaire, 1978.

GRACIA ALONSO, Francisco, y FULLOLA PERICOT, Josep. El sueño de una generación: el crucero universitario por el Mediterráneo de 1933. Barcelona: Edicions de la Universitat de Barcelona, 2006.

GUERRERO, Gustavo. Historia de un encargo: «La catira» de Camilo José Cela. Barcelona: Anagrama, 2008.

GULLÓN, Ricardo. Conversaciones con Juan Ramón Jiménez. Sevilla: Sivilina, 2008.

GUTIÉRREZ-SOLANA, José. La España negra. Granada: Editorial Comares, 2000.

ORTEGA CANTERO, Nicolás. «La experiencia viajera en la Institución Libre de Enseñanza». En: VV.AA. Viajeros y paisajes. Madrid: Alianza Universidad, 1988.

ORTEGA Y GASSET, José. Obras Completas I (1902-1916). Madrid: Revista de Occidente, 1946.

PACK, Sasha D. La invasión pacífica. Los turistas y la España de Franco, Marí, Ana (trad.). Madrid: Taurus, 2009.

PASQUALI, Adrien. Le tour des horizons. Critique et récits de voyages. París: Klincksieck, 1994.

PEMÁN, José María. Narraciones y ensayos. Obras Completas II. Madrid: Escelicer, 1947.

—. Doctrina y oratoria. Obras Completas V. Madrid: Escelicer, 1947. 
SICOT, Bernard. Exilio, memoria e historia en la poesía de Luis Cernuda. México: Fondo de Cultura Económica, 2003.

UNAMUNO, Miguel de. Obras completas I. Paisajes y ensayos. Madrid: Escelicer, 1966.

VERHAEREN, Emile, y REGOYOS, Darío de. La España negra. Madrid: Taurus, 1963.

Fecha de recepción: 17 de junio de 2010

Fecha de aceptación: 16 de diciembre de 2010 